\title{
The robust solution for epidemiology
}

\author{
Paul T E Cusack* \\ Independent Researcher, Canada
}

\section{Abstract}

Based on my study of cholera and mental illness, in particular, schizophrenia, I've discovered some mathematical laws that to apply to epidemiology. It is the familiar "Robust Solution" that I've developed in other papers on physics and economics. The same math applied in the study of the transmission and termination of contagious disease What I provide here is mathematics from the Robust solution that applies to epidemiology.

\section{Introduction}

We consider conditional probability, the Gaussian distribution, Overcrowding, resistance to disease, the golden mean below. We develop a basic law of contagion.

\section{Conditional probability}

$$
\begin{aligned}
& \operatorname{Pr}[A / B]=\operatorname{Pr}[A \cap B\} / \operatorname{Pr}[A] \\
& =1-\sin \theta \\
& =0.1585 \\
& \mathrm{E}=1-\mathrm{F} \\
& =1-\sin \theta \\
& =0.1585 \\
& E=e^{-t}
\end{aligned}
$$

\section{Gaussian distribution}

$$
\begin{aligned}
& \Phi=1 / \sqrt{ }(2 \pi) e^{-t^{2} / 2} \\
& =\mathrm{E}=1-\mathrm{F} \\
& e^{-t} \alpha e^{-t^{2} / 2}=1-F \\
& e^{-t}-e^{-t^{2} / 2}=-1 \\
& -t+t^{2} / 2+0=0 \\
& t^{2} / 2-t=0 \\
& \mathrm{t}=0,2 \\
& 2^{2}-2-1=E=1 \\
& \mathrm{E}=1-\mathrm{F} \\
& 1=1-\mathrm{F} \\
& \mathrm{F}=0 \\
& \mathrm{~F}=\sin \theta \\
& \theta=0, \pi, 2 \pi \\
& E=e^{-\pi}=4.32 \quad 43.2 \% \\
& 1-43.2=56.8=1 / \sqrt{ } \pi
\end{aligned}
$$

$$
t^{2} / 2-1 / t(1 / \sqrt{ } \pi)^{2}-(1 / \sqrt{ } \pi)=0.1592 \sim 1-\sin \theta=E
$$

Overcrowding resistance to disease, and the Golden Mean parabola:

\section{Moment=Fd}

$\mathrm{Fd}=1-\sin 1$

$8 / 3(\mathrm{~d})=0.1585$

$\sin \theta d=c u z=(\pi-e)=0.4233$

$\sin t(d)=c u z$

$\sin \mathrm{t}=\mathrm{F}$

$=8 / 3$

$\mathrm{d}=\mathrm{cuz}^{\star} \mathrm{F}=0.4233 / 23.667=1 / 2 \mathrm{Pi}=1$ radian $=0.40 \%$ of a cycle (April -September Season)

\section{Cusack's Contagion Law}

Infected ${ }^{*} d=$ Resistance to infection

Increase distance $==>$ Increase resistance

Decrease distance $==>$ decrease resistance

Derivatives:

Infected $=\mathrm{R}_{\mathrm{d}}(1 / \mathrm{d})$

Infected ' $=\mathrm{R}_{\mathrm{d}}\left(-1 / 2 \mathrm{~d}^{2}\right)$

Let $\mathrm{d}=0$

Infected' $=0$

$2 \mathrm{t}-1=0$

Correspondence to: Paul T E Cusack, BScE, DULE, 1641 Sandy point Rd, Saint John, NB E2K 5E8, Canada. Tel: (506) 214-3313; E-mail: St-michael@hotmail.com

Key words: epidemiology, Gaussian distribution

Received: February 03, 2017; Accepted: February 17, 2017; Published: February 21,2017 
$\mathrm{t}=1 / 2==>$ minimum $\mathrm{E}$ of the golden mean parabola.

$\mathrm{E}=-1.25=10 / 8=5 / 4$

$1 / \operatorname{Sin} \mathrm{t}=5 / 4$

$\mathrm{t}=52.1$ degrees

And,

$1 / \mathrm{F}=1 /(8 / 3)=0.375$

$\left.0.375)^{\wedge}-1\right)(5 / 4)=46.8 \%$ cg $46.25 \%=\operatorname{Pr}[$ Cholera $\&$ Mental illness $]$

$\mathrm{E}=$ Work $^{\star} \mathrm{t}$

$=\mathrm{Fd}^{*} \mathrm{t}$

$-1.25=(8 / 3)(\mathrm{d})(1)$

$\mathrm{d}=46.8$ cf $46.25 \%$

And $\mathrm{E}=(\pi-\mathrm{e})$

$(\mathrm{t}-\mathrm{E})=\mathrm{E}$

$(\mathrm{t}-1 / \mathrm{t})=\mathrm{E}$

$\left[\left(\mathrm{t}^{2}-1\right] / \mathrm{t}=\mathrm{E}\right.$

$\mathrm{E}=1$

$\mathrm{t}^{2}-\mathrm{t}=1$

$\mathrm{t}^{2}-\mathrm{t}-1=0$

Golden Mean

\section{Probabilities and the Contagion}

$\operatorname{Pr}[A \cup B] 1-\sin 1=0.1585$

$\operatorname{Pr}\{A]+\operatorname{Pr}[B]-\operatorname{Pr}[A \cap B]=15.85 \%$

$\operatorname{Pr}[$ Having Mental illness] $+\operatorname{Pr}$ (Having Cholera]- $\operatorname{Pr}[\mathrm{A} \cap \mathrm{B}]$ $=$ Energy of the Contagion
$90 / 30,000+5000 / 30,000-\operatorname{Pr}\{A \cap B]=15.85 \%$

$0.3 \%+16.67 \%-\operatorname{Pr}[A \cap B]=15.85 \%$

$\operatorname{Pr}\{A \cap B]=1.12 \%=$ Probability of having Sz.

$\operatorname{Pr}\{A / B]=\operatorname{Pr}[A \cap B] / \operatorname{Pr}\{A]$

$1-\operatorname{Pr}[A \cap B]=84.15 \%$

$\operatorname{Pr}[A \cap B]=15.85 \%$

$\operatorname{Pr}[A / B] * \operatorname{Pr}[A]=15.85 \%$

$\operatorname{Pr}[A / B] * 0.3 \%=15.85 \%$

$\operatorname{Pr}[A / B]=52.83 \%$

$1-52.83 \%=47.17 \%$ cf $46.3 \%$ for cholera

Now,

$\operatorname{Pr}[$ Dying from Cholera / Mental illness $]=$

$=37.85 \%-34.6 \%=3.25 \% \sim 3.3 \%$

$\operatorname{Pr}[A / B]=37.85 \%$

\section{Conclusion}

So we see that the Robust solution mathematics applies to Epidemiology as well as it applies to any two pole problem, under which lies the Gaussian distribution.

\section{References}

1. Somerville GF (1854) Saint John and Portland cholera Deaths. Saint John, NB

2. Bislon G (1854) The Cholera Epidemic in Saint John, NB Acadiensis

3. Weiss NA (2008) Introductory Statistics, 8th Ed. Pearson, Addison Wesley, USA.

4. Cusack P (2015) Sz and Its Cause., LULU.

5. Cholera and Mental Illness, Mental health, Family Medicine

6. Iron, Cholera, and Mental illness in Nineteenth-Century Saint John. Clinical Investigation and Medical Research, OMI.

Copyright: $@ 2017$ Cusack PTE. This is an open-access article distributed under the terms of the Creative Commons Attribution License, which permits unrestricted use, distribution, and reproduction in any medium, provided the original author and source are credited. 